\title{
Exact and inexact differentials in the early development of mechanics and thermodynamics
}

\author{
Mário J. de Oliveira*1] \\ ${ }^{1}$ Universidade de São Paulo, Instituto de Física, São Paulo, SP, Brasil
}

Received on July 31, 2019. Revised on October 8, 2019. Accepted on October 13, 2019.

\begin{abstract}
We give an account and a critical analysis of the use of exact and inexact differentials in the early development of mechanics and thermodynamics, and the emergence of differential calculus and how it was applied to solve some mechanical problems, such as those related to the cycloidal pendulum. The Lagrange equations of motions are presented in the form they were originally obtained in terms of differentials from the principle of virtual work. The derivation of the conservation of energy in differential form as obtained originally by Clausius from the equivalence of heat and work is also examined.
\end{abstract}

Keywords: differential, differential calculus, analytical mechanics, thermodynamics.

\section{Introduction}

It is usual to formulate the basic equations of thermodynamics in terms of differentials. The conservation of energy is written as

$$
d U=d Q-d W
$$

where $d U, d Q$, and $d W$ are the differentials of the internal energy, the heat absorbed and the work performed by a system. The conservation of energy is expressed by equation (1) as long as $d U$ is an exact differential, a key feature that is not explicitly stated but is tacitly understood. An exact differential such as $d U$ means that there exists a state function $U$ such that its differential is $d U$. An inexact differential such as $d Q$ and $d W$, does not hold this property. It should be pointed out that some authors use a distinction notation to refer do to an inexact differential. One of them is to cross the differential, a procedure that becomes quite confusing and useless.

The differential of the work $d W$ is written in terms of exact differential. In the case of mechanical work, for instance, $d W=p d V$ where $p$ is the pressure and $d V$ is the differential of the volume $V$. In an analogous way the differential of heat can also be written in terms of an exact differential. If a system is in thermodynamic equilibrium than $d Q / T$ is the exact differential $d S$ of the entropy $S$. This result allows us to write the relation $d Q=T d S$ between heat and entropy, which should be understood as valid as long as the system is in thermodynamic equilibrium.

Differentials appeared with the invention of calculus in the second half of the seventeenth century. The differential $d x$ was understood as a small increment of the

*Correspondence email address: oliveira@if.usp.br variable $x$. If another variable $y$ depends on the independent variable $x$, then the resulting increment $d y$ of $y$ is its differential. The quotient of these two differentials, $d y / d x$, was interpreted geometrically by Leibniz as the ratio of the ordinate $y$ of a point on a curve and the length of the subtangent associated to this point.

The formulation of thermodynamics in terms of differentials seemed to be a natural consequence of the application of differential calculus at the time of the emergence of thermodynamics around the middle of the eighteenth century [1]. Clausius formulated the laws of thermodynamics by the use of differentials $[2]$. The same can be said about the development of mechanics in the eighteenth century [3], particularly the formulation of analytical mechanics by Lagrange [4].

Here, we give an account and an analysis of the development of the concept of differential and its application during the early development of mechanics and thermodynamics. Our presentation starts with the introduction of the differential and integral calculus by Newton and Leibniz. Then we analyze the developments of the basic rules of differential calculus and the concepts of integrating factor, exact and inexact differentials. Next we show how the application of differential calculus was used to solve mechanical problems such as the brachistochone problem. In the second part, we analyze how the differentials were used by Lagrange in his analytical mechanics and by Clausius in his development of thermodynamics.

As a generic procedure of our exposition we try to use the terminology and notation originally employed by the authors. However, for the sake of a better understanding by the reader, sometimes we use the modern terminology and notation as long as they do not modify the original meaning of the concept being described. 


\section{Differential calculus}

\subsection{Basic rules}

The differential and integral calculus was invented independently by Newton and Leibniz in the second half of the seventeenth century $5-10$. They provided algorithm procedures, including the basic rules of the calculus, which were essential for the development of the concept of derivative and integral [5]. Newton developed the calculus in the years $1665-1666$ but his first publication on the subject, which was written in 1676, appeared only in 1704 as a mathematical appendix to his Opticks, although portions of his Principia, published in 1687, contained some of his achievements on calculus 11]. Leibniz developed the subject during the years 1673-1676 and the accounts of his findings were published in two papers 8 . The first, in 1684, on differential calculus [12], the second, in 1686, on integral calculus 13. Leibniz also created a convenient notation, without which the calculus could not achieve its fundamental role in mathematics [7].

The differential calculus may have its origin in geometric problems such as that of drawing tangents or of locating the maxima and minima of curves [14]. Its origins is found within the domain of analytic geometry where geometry is studied by means of a coordinate system. A curve drawn in a plane is represented in analytic geometry by an equation involving two algebraic variable, the abscissa and the ordinate, which in Cartesian geometry are understood as the distances of a point on the curve from two orthogonal axes. In his first publication on the differential calculus of 1684 12, Leibniz denoted the differential of a variable $x$ by $d x$, the same notation that is used today. It was interpreted as a small increment in the abscissa $x$, which implies an increment, or a differential, $d y$ in the ordinate $y$, as represented geometrically in figure 1. Leibniz states that the ratio between $d y$ and $d x$ equals the ratio between the ordinate and the subtangent.

The development of Leibniz calculus during the first decades after its discovery was accomplished mainly by Jacob Bernoulli and Johann Bernoulli, and the first book on the subject was published in 1696 by l'Hôpital [15, based on the works of Leibniz, Jacob Bernoulli, and specially on those of Johann Bernoulli. It contained the basic rules of the differential calculus, and included procedures for determining tangents, maxima and minima, inflexion points, involutes and evolutes, and caustics of plane curves.

L'Hôpital starts his exposition on differential calculus by giving the basic rules of the subject, such as the differential of the sum of two variables $z=x+y$,

$$
d z=d x+d y
$$

of the product of two variables $z=x y$,

$$
d z=y d x+x d y
$$

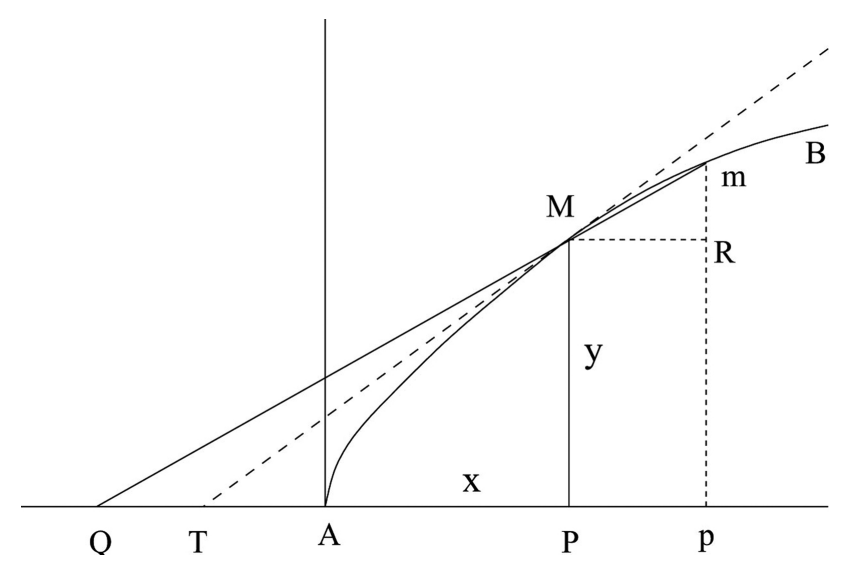

Figure 1: The dashed line TM is the tangent to the curve $A M B$ at the point $M$ and $Q M m$ is a straight line that crosses the curve at the same point. The segments AP and MP are the abscissa $x$ and ordinate $y$ of the point $\mathrm{M}$ whereas $\mathrm{Pp}$ and $\mathrm{Rm}$ are the differentials $d x$ and $d y$. The ratio between $d y$ and $d x$ equals the ratio between the ordinate MP and the subtangent TP. The figure is based on figures 1 and 3 of reference [15].

of the quotient of two variables $z=x / y$,

$$
d z=\frac{y d x-x d y}{y^{2}},
$$

of a power $z=x^{m}$,

$$
d z=m x^{m-1} d x
$$

where $m$ is a rational number, positive or negative.

The determination of the tangents of curves, requires the calculation of the subtangent, the length of the segment TP of figure 1 If $\operatorname{Pp}(d x)$ is small, then $\mathrm{Rm}(d y)$ is also small, the triangles MRm and TPM become similar and as a consequence the length $t$ of the subangent TP is to $y$ as $d x$ is to $d y$,

$$
\frac{y}{t}=\frac{d y}{d x}
$$

The right-hand side is understood as the quotient between the two differentials. In fact, this is the expression used by Leibniz to define $d y$ by introducing $d x$ as an arbitrary finite interval $[12$. To find the maximum value of the ordinate of a curve it suffices to set the expression of $d y$ obtained for a given curve equal to zero. L'Hôpital gives the following example,

$$
x^{3}+y^{3}=a x y,
$$

which described a certain plane curve. According to the basic rules,

$$
d y=\frac{a y d x-3 x^{2} d x}{3 y^{2}-a x} .
$$

Setting this expression equal to zero he finds $y=3 x^{2} / a$ which replaced in equation $(7)$, gives $x=(a / 3) \sqrt[3]{2}$, the value of $x$ at which the curve has its maximum value. 
The determination of inflexion points of curves was also considered by l'Hôpital. In this case it is necessary to find the second differential $d^{2} y$ of the ordinate $y$, understood as the differential of the differential $d y$ and thus holding the same rules of the first differential. As an example, he considers the curve described by

$$
y=\frac{a x^{2}}{x^{2}+a^{2}}
$$

From this expression, one finds

$$
d y=\frac{2 a^{3} x d x}{\left(x^{2}+a^{2}\right)^{2}}
$$

The second differential is also determined by the basic rules, yielding

$$
d^{2} y=\frac{\left(2 a^{5}-6 a^{3} x^{2}\right) d x^{2}}{\left(x^{2}+a^{2}\right)^{3}}
$$

Setting the numerator equal to zero, he finds the values $x=a / \sqrt{3}$ for the location of the inflexion point. The corresponding ordinate is $y=a / 4$.

\subsection{Integrating factor}

The differential calculus was by its origin a natural method for solving geometric problems, the results being expressed in terms of algebraic expressions 5. In this sense it was a method to solve problems in analytic geometry. This changed with Euler, who made the subject a theory of functions without the need of a connection with geometry [5]. Notice however that, for Euler the meaning of function was a formal expression in terms of variables and constants and did not have the present meaning of a conceptual relationship between a quantity and an independent variable 5 .

In a paper presented in 1728, published in 1732 [16], concerning a method to reduce second order into first order differential equations, Euler introduces the integrating factor. As an example, he considers the following differential equation

$$
d z+\frac{2 z d t}{t-1}+\frac{d t}{t^{2}-t}=0
$$

To solve equation (12), Euler multiplies the equation by the integrating factor $(t-1)^{2}$, obtaining

$$
(t-1)^{2} d z+2 z(t-1) d t+(t-1) \frac{d t}{t}=0 .
$$

The last term is the differential of $t-\ln t$, and the first two terms is identified as the differential of $(t-1)^{2} z$. Thus the integration gives

$$
(t-1)^{2} z+t-\ln t=a,
$$

where $a$ is a constant.
Equation 12 is of the general type

$$
d z+z P d t+Q d t=0
$$

where $P$ and $Q$ are functions of $t$ and can be solved in like manner. According to Euler, the integrating factor is

$$
x=e^{\int P d t} .
$$

Multiplying equation (15) by $x$, and taking into account that $d x=x P d t$, one finds

$$
x d z+z d x+x Q d t=0,
$$

which after integration gives

$$
x z+\int x Q d t=a .
$$

This equation gives $z$ as a function of $t$.

\subsection{Complete differential}

In another paper, presented in 1734 and published in 1740 17, Euler considers a function $V$ of two variables $x$ and $y$. He writes the differential of $V$ as

$$
d V=P d x+Q d y
$$

where the first term is obtained by considering $y$ constant and the second by considering $x$ constant. Writing $d P=$ $p d x+r d y$ and $d Q=q d x+s d y$, Euler argues that $q=r$ 17. Later, he introduces the notation 18

$$
r=\left(\frac{d P}{d y}\right), \quad q=\left(\frac{d Q}{d x}\right),
$$

and writes the equality $q=r$ as

$$
\left(\frac{d P}{d y}\right)=\left(\frac{d Q}{d x}\right) .
$$

Euler warns that not all expressions of the type (19) are differential of a certain function of $x$ and $y$. If the relation (21) does not hold, no function of $x$ and $y$ exists such that its differential is $P d x+Q d y$. An example given by Euler is $y x d x+x^{2} d y$.

In a paper published in 1742 [19], Clairaut shows that, if $A d x+B d y$ represents the differential of a quantity dependent on $x$ and $y$, then

$$
\frac{d A}{d y}=\frac{d B}{d x},
$$

which is the condition 21 found by Euler. According to Clairaut, this condition allows us to known if $A d x+B d y$ is the differential of a certain quantity in two variables. If the condition is fulfilled, Clairaut calls $A d x+B d y$ a complete differential. As an example of a complete differential he gives

$$
\frac{y d x-x d y}{x^{2}+y^{2}}
$$


In his book on the figure of the earth published in 1743 [20], Clairaut states that the integral of a complete differential is a function of $x$ and $y$ and he gives the following two examples

$$
y d x+x d y, \quad \frac{y d x+x d y}{2 \sqrt{a^{2}+x y}},
$$

which have as integrals $x y$ and $\sqrt{a^{2}+x y}$, respectively.

To integrate a complete differential $A d x+B d y$, Clairaut uses the following scheme 19. One integrates one of the members, say $A d x$ supposing $y$ constant, and to the result one adds a quantity $C$ that depends only on $y$. Next one calculates the differential of the sum, keeping $x$ constant, and subtract $B d y$. The result is either zero or is the differential of $C$, which can thus be integrated and added to $\int A d x$.

If the expression $M d x+N d y$ is not a complete differential, Clairaut explains that it is possible to render a complete differential by multiplying the expression by a factor $\mu$ dependent on $x$ and $y$ [19]. Assuming that $\mu M d x+\mu N d y$ is a complete differential, it follows from the condition 22 that

$$
\frac{d(\mu M)}{d y}=\frac{d(\mu N)}{d x},
$$

an equation to be solved for finding $\mu$.

\subsection{Derivative}

The differential and integral calculus as invented by Newton and Leibniz and developed by their immediate successors was very successful, becoming the mathematical basis of physical theories such was the case of Newtonian mechanics. However, criticisms were raised concerning its foundations particularly the employment of infinitely small quantities [5] as occurs in the differential quotient $d y / d x$. If the differential is a vanishing quantity then the differential quotient would result in an indetermination ratio of the type zero over zero. On the other hand, the rules of the calculus established by Newton and Leibniz give well defined results for the differential quotient. A crucial contribution toward the clarification of this problem was presented by d'Alembert in his article on differential contained in the Encyclopédie, where he explains how to find the differential quotient by using the concept of limit 21.

As we have seen above the differential quotient is equal to the ratio of the ordinate MP of a point $\mathrm{M}$ of a curve and the subtangent TP as shown in figure 1. D'Alembert shows this result by considering a straight line QMm, which crosses the curve AMB at the point M. As the point $\mathrm{m}$ approaches $\mathrm{M}$, the point $\mathrm{Q}$ moves toward the point $\mathrm{T}$ and as a consequence the ratio $\mathrm{mR} / \mathrm{MR}$, which equals the ratio $\mathrm{MP} / \mathrm{QT}$, aproaches the ratio $\mathrm{MP} / \mathrm{TP}$ of the ordinate and the subtangent. D'Alembert gives the example of a curve described in algebraic terms by $a x=y^{2}$. Denoting $\mathrm{mR}$ by $z$ and $\mathrm{MR}$ by $u$, the ratio
$\mathrm{mR} / \mathrm{MR}$ is $a /(2 y+z)$ which, as $z$ decreases, approaches the limit $a / 2 y$. According to the rules of the calculus, $a d x=2 y d y$, which gives also $d y / d x=a / 2 y$.

Up to the last decades of the eighteenth century the differential calculus was centered on differentials. As we have seen above, the differential equations were written in terms of differentials. This state of affairs changed with Lagrange. In his paper on differential and integral calculus [22], published in 1774, and on his theory of analytical functions 23, published in 1797, the prominent role was played by the derivative, or derived function in the terminology introduced by as Lagrange. He also introduced the notation $f x$ for a function of a variable $x$, without parentheses, but we will write $f(x)$. However, he used parentheses in the case $f(x+a)$.

Wishing to avoid differentials, Lagrange did not use the ratio of differentials as the definition of derivative. Instead he based his definition on the Taylor series. Taylor wrote the series that bears his name by considering two quantities $z$ and $x$. When $z$ increases to become $z+v$, the quantity $x$ becomes

$$
x+\dot{x} \frac{v}{1 \dot{z}}+\ddot{x} \frac{v^{2}}{1.2 \dot{z}^{2}}+\dddot{x} \frac{v^{3}}{1.2 .3 \dot{z}^{3}}+\text { etc. }
$$

Taylor published this result in $1715[24]$ using the notation and terminology introduced by Newton. A quantity $x$ is called a fluent and its rate of change, denoted by $\dot{x}$, is called the fluxion of $x$. Thus, in expression (26), the ratio between $\dot{x}$ and $\dot{z}$ is the derivative of $x$ with respect to $z$; the ratio between $\ddot{x}$ and $\dot{z}^{2}$ is the second derivative; and so on.

If one wishes to construct a Taylor series for a certain function, one needs to know a priori the derivatives. However, the series can be obtained by other means without reference to derivatives. If this alternative procedure is employed, the derivatives could be obtained by comparing with the Taylor series. This was the reasoning of Lagrange, who considered the Taylor series as a way to define the derivative of a function $\sqrt[5,9]{9}$. He writes the fraction

$$
P=\frac{f(x+a)-f(x)}{a},
$$

and then sets $a=0$ after performing the subtraction in the numerator by the use of the power expansion in the increment $a$ 22, 23]. He denotes the result $f^{\prime} x$, but we will write $f^{\prime}(x)$, and calls it the derived function of $f(x)$. As an example, Lagrange considers $f(x)=\sqrt{x}$, from which

$$
P=\frac{\sqrt{x+a}-\sqrt{x}}{a}=\frac{1}{\sqrt{x+a}+\sqrt{x}} .
$$

Setting $a=0$, one finds $f^{\prime}(x)=1 / 2 \sqrt{x}$.

The definition of derivative given by Lagrange was based on the assumption that a function $f(x)$ could be expanded in power series in the increment of $x$. Cauchy noted that this is not always possible. He then advanced a definition based on the concept of limit 9. D'Alembert 
had already proposed this procedure but it lacked a proper definition of limit, a concept that was given by Cauchy in the following terms [25]:

When the values successively assigned to the same variable approach indefinitely to a fixed value, so as to end by differing from it as little as desired, the latter is called the limit of all the others.

His definition of derivative becomes the limit of the quotient 28) when $a$ approaches zero. This verbal definition, Cauchy translated into the precise language of deltas, epsilons and inequalities 26.

Cauchy also provided a meaning to the differential $d y$ of a function $y=f(x)$ 25. The differential of the independent variable $d x$ is a finite constant and

$$
d y=f^{\prime}(x) d x
$$

According to Cauchy, this relation is the reason to call $f^{\prime}(x)$ the differential coefficient [25], a terminology that was introduced by Lacroix [5].

\subsection{The concept of function}

The word function was used by Leibniz as the meaning of any quantity connected to a curve such as the locus, the slope, or the radius of curvature 14. Johann Bernoulli and Euler regarded a function as an expression or a formula involving variables and constants, which is the concept usually held by the students of elementary mathematics courses [14. The same concept of function was used by Lagrange. In his treatise on analytical functions, he writes 23 :

We call function of one or more quantities, any expression of calculus in which these quantities enter in any manner, mixed or not with other quantities that we consider as having given and invariable values, while the quantities of the function can receive all the possible values.

Fourier, in his investigation on the equation of heat flows, departs from the notion of function as an analytic expression and approaches the modern concept of function. In his treatise on the theory of heat, where he develops the series that bears his name, he writes [27]:

In general, the function $f x$ represents a sequence of values, or ordinates, each of which is arbitrary. The abscissa $x$ can receive an infinity of values, there is an equal number of ordinates $f x$. All have actual numeric values, either positive, or negative, or zero. It is not supposed that these ordinates are subject to a common law; they succeed one another in whatever manner, and each of them is given as it were a single quantity.
Dirichlet improved the definition given by Fourier arriving at a formulation that was based on the idea of function as a relationship between two variables [14,28. It can be stated as follows: if $x$ and $y$ be two variables such that for each value assigned to $x$ there corresponds, by some rule which need not be an analytical expression, then $y$ is a function of $x$.

\section{Mechanics}

\subsection{Laws of motion}

The fundamental laws of motion were established by Newton in his treatise on mechanics, known as the Principia, published in 1687 [29]. A translation from the original in Latin was published later in 1729 [30]. Newton was not the first to formulate laws of motion. Prior to Newton, Galileo had formulated the law of inertia, which is one the fundamental laws of Newtonian mechanics, and also studied the motion of falling bodies, the trajectory of projectiles and the oscillations of a pendulum.

In his Dialogues Concerning two New Sciences, Galileo describes his observations of oscillations in the following terms 31, 32]:

Thousands of times I have observed vibrations especially in churches where lamps, suspended by long cords, had been inadvertently set into motion.

These occurrences might have stimulated him to study the oscillations of a pendulum. Galileo found that the period of oscillations of a simple pendulum is proportional to the length of the pendulum. He might have considered that the period is independent of the amplitude of oscillations but there is no mention concerning this issue on his writings 33 .

In his treatise on the pendulum clock, published in 1673, Huygens showed that a cycloidal pendulum is isochronous, that is, the period is independent of its amplitude [34]. In contrast to a simple pendulum, which follows a circular path, a cycloidal pendulum follows a cycloid, a curve traced by a point on the edge of a circle rolling without sliding along a straight line, as shown in figure 2. When the point $\mathrm{K}$ goes into the point $\mathrm{M}$, the disk rotates by an angle LOK so that MN equals the displacement of the center of the disk, which is the arc LK, plus LN, which gives ML equals the arc LK. Considering that $\mathrm{CN}$ is equal to the semi-circumference, it follows that $\mathrm{CM}$ is equal to the arc LG minus LN, which defines geometrically the cycloid. In modern notation and in parametric form the cycloid is described by

$$
x=r(1-\cos \theta), \quad y=r(\theta-\sin \theta),
$$

where $x$ and $y$ denotes the distances BM and CM, $\theta$ is the angle LOG and $r$ is the radius of the disk. Huygens demonstrated the isochronous properties of a cycloid by 


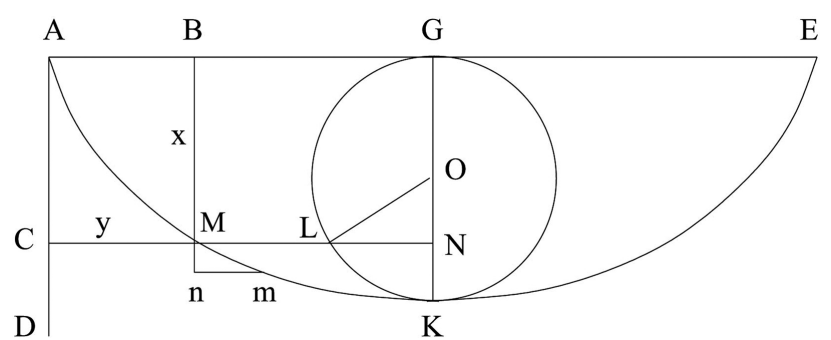

Figure 2: The curve AMKE is a cycloid generated by a point on the edge of the disk which rolls without sliding along the straight line AGE. The segments $C M$ and BM are the abscissa $y$ and ordinate $x$ of point $\mathrm{M}$ whereas $\mathrm{nm}$ and $\mathrm{Mn}$ are the differentials $d y$ and $d x$. The figure is based on figure 1 of reference [36].

showing that it is a tautochrone, a curve holding the property that the time it takes for a body to descend to the lowest point is the same regardless of the starting point.

The cycloid is also the solution of the brachistochrone problem, posed by Johann Bernoulli in 1696 [35. The problem is to find the curve which gives the fastest descent of a sliding body moving between two points that are not in the same vertical line. The solution given by Johann Bernoulli himself was based on the Fermat principle of the least time, which he adapted to his mechanical problem [36]. Accordingly, he used the sine law which is obtained from this principle. This law states that the sine of the angle of inclination of the curve at a certain point with respect to the vertical is proportional to the velocity. Referring to the figure 2 it is the ratio of the differentials $d y=\mathrm{nm}$ and $d s=\mathrm{Mm}$, which is thus proportional to the velocity $v$, that is,

$$
\frac{d y}{v}=\frac{d s}{a}
$$

or $a d y=v d s$, which after squaring gives $a^{2} d y^{2}=v^{2} d s^{2}$. Taking into account that $d s^{2}=d x^{2}+d y^{2}$, where $d x=\mathrm{Mn}$, one finds $\left(a^{2}-v^{2}\right) d y^{2}=v^{2} d x^{2}$ from which follows the equation

$$
d y=\frac{v d x}{\sqrt{a^{2}-v^{2}}} .
$$

To relate velocity and position, Johann Bernoulli uses the relation found by Galileo in his investigation on the fall of bodies according to which the velocity is proportional to the square root of the altitude traversed from the rest, $v=\sqrt{a x}$. Employing this relation, Johann Bernoulli reaches the differential equation

$$
d y=d x \sqrt{\frac{x}{a-x}} .
$$

To shown that this equation describes a cycloid, he writes this equation in the equivalent form

$$
d y=d x \frac{x}{\sqrt{a x-x^{2}}}=\frac{a d x}{2 \sqrt{a x-x^{2}}}-\frac{a d x-2 x d x}{2 \sqrt{a x-x^{2}}} .
$$

The integral of the second term is $\sqrt{a x-x^{2}}$, which is LN of figure 2, and the integral of the first term is the

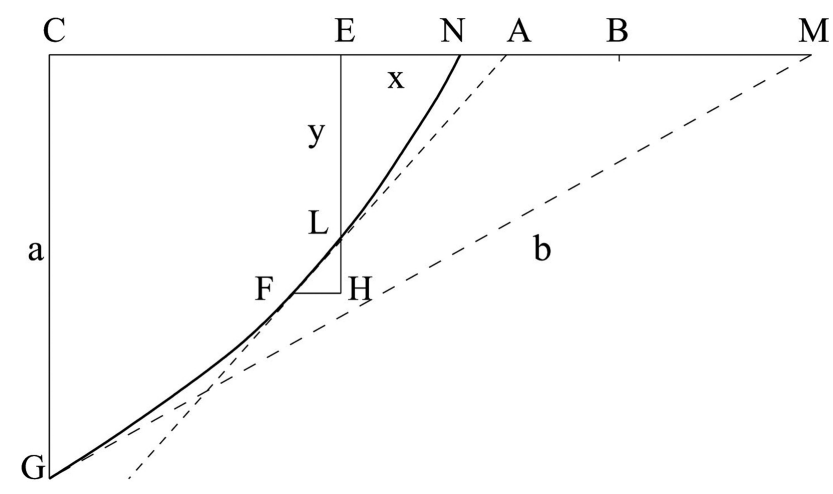

Figure 3: The curve GLA is a semi-cubic parabola. The dashed lines are tangents to the curve GLA at the points $L$ and $G$. FH and $\mathrm{LH}$ are the differentials $d x$ and $d y$ and $\mathrm{BE}$ and $\mathrm{EL}$ are the abscissa $x$ and ordinate $y$ of the point $L$. The figure is based on figure 1 of reference [38].

length of the arc GL. The total integral $y=$ GL - LN, which is the statement that AMK is cycloid as we have seen above.

In 1687, Leibniz posed the following problem. Find a line of descent, in which a heavy body descends uniformly, and also approaches the horizontal line in equal time, that is, with a constant vertical velocity [37. The solution is a semi-cubic parabola and a solution was given by Jacob Bernoulli in a paper published in 1690 38. A differential equation describing the curve was set up by Jacob Bernoulli as follows. The straight lines AL and $\mathrm{MG}$ are tangents to the points $\mathrm{L}$ and $\mathrm{G}$ of the curve NLG shown in figure 3 The ratio of $\mathrm{LH}=d y$ and $\mathrm{FL}=\sqrt{d x^{2}+d y^{2}}$ is equal to the ratio of the vertical component of the velocity $v_{y}$ and the velocity $v$ at the point L,

$$
\frac{d y}{\sqrt{d y^{2}+d x^{2}}}=\frac{v_{y}}{v} .
$$

A similar relation can be written for the point $G$,

$$
\frac{a}{b}=\frac{V_{y}}{V}
$$

Considering that the vertical velocity is the same, $v_{y}=$ $V_{y}$, we find the following relation

$$
\frac{b}{a} \frac{\sqrt{d y^{2}+d x^{2}}}{d y}=\frac{v}{V}
$$

Jacob Bernoulli uses the Galileo result according to which the velocity is proportional to the square root of the vertical altitude, to reach the result

$$
\frac{a}{b} \frac{\sqrt{d y^{2}+d x^{2}}}{d y}=\frac{\sqrt{y}}{\sqrt{a}}
$$

. After squaring,

$$
a^{3}\left(d y^{2}+d x^{2}\right)=b^{2} y d x^{2}
$$

the following differential equation is found

$$
d x \sqrt{a^{3}}=d y \sqrt{b^{2} y-a^{3}},
$$


that describes the desired curve.

The integral found by Bernoulli is

$$
x=\frac{2}{3} \frac{b^{2} y-a^{3}}{b^{2} \sqrt{a^{3}}} \sqrt{b^{2} y-a^{3}} .
$$

Replacing $y-a^{3} / b^{2}=z$,

$$
x^{2}=\frac{4 b^{2}}{9 a^{3}} z^{3},
$$

which describes a semi-cubic parabola.

\section{Analytical mechanics}

\subsection{Principle of d'Alembert}

Analytical mechanics was the name that Lagrange chose for his book on mechanics to emphasize the analytical framework on which rests his treatise on the subject [4]. He stressed his point of view by remarking that [4]

\begin{abstract}
No figures will be found in this work. The methods that expose in it require neither constructions nor geometrical or mechanical reasonings, but only the algebraic operations inherent to a regular and uniform process. Those who love analysis will, with joy, see mechanics become a new branch, and I am grateful that I have extended its domain.
\end{abstract}

The analytical mechanics of Lagrange is founded on the principle of virtual work, which was developed by Johann Bernoulli, in the static version, and by d'Alembert, in the dynamic version.

The principle of virtual work stated by Johann Bernoulli generalized the previous formulations given by his predecessor 3]. The principle gives the condition for the static equilibrium of a mechanical system acted by several forces. In a letter written in 1717 to Varignon, which was reproduced in the treatise of Varignon on statics 39], Johann Bernoulli states the principle of virtual work, which he calls principle of virtual velocities, as follows:

In all equilibrium of any forces, in whatever way they are applied, and in whatever direction that they act on each other, indirectly or directly, the sum of the positive energies will be equal to the sum of the negative energies taken positively.

In this statement, energy means the product of the magnitude of a force by a small displacement of the point acted by the force. The small displacement is parallel to the force, being positive if in the direction of the force and negative in the opposite direction. It should be noted that the small displacements Johann Bernoulli called virtual velocities.

In his treatise on analytical mechanics, Lagrange adopts the principle of virtual work, as a fundamental principle of statics. He writes the principle in the analytical form as

$$
\sum_{i} Q_{i} d q_{i}=0
$$

where $Q_{i}$ are forces acting along the lines that define center of foces, and the differentials $q_{i}$ are small displacements along the lines of forces, described by the variables $q_{i}$. Lagrange states that the equation 43 is the condition of equilibrium of a system of forces. It should be remarked that forces of reaction, which occurs for instance when a body rests on a surface, do not enter this equation because their works perform no work.

Lagrange supposes next that the expression in the lefthand side of 43 is an exact differential $d \Phi$ of a function $\Phi$, which means that $\Phi$ is a function of the variables $q_{i}$. In modern terms, Lagrange assumes that the forces are conservative, $\Phi$ being understood as the potential energy. The condition for equilibrium becomes $d \Phi=0$, which means that the systems is configured in such as way that $\Phi$ is a maximum or a minimum. Lagrange shows furthermore that if the function is a minimum then the equilibrium is stable and the system will display small oscillations. If on the contrary the function is a maximum, the equilibrium is unstable and, being once disturbed, the system will depart from equilibrium.

The static principle of virtual work of Johann Bernoulli was extended to dynamic problems by d'Alembert [40]. According to d'Alembert, in dynamic problems one should take into account the inertial forces in addition to the actual forces. An analytical expression of the d'Alembert principle was given by Lagrange, and was obtained as follows. Lagrange argues that, the laws of motion of a body will be reduced to the laws of equilibrium if, following d'Alembert, one includes the inertial force, which is the mass of the body multiplied by the acceleration [4].

If we denote by $x_{i}$ a Cartesian coordinate of a body of mass $m$, the work of the inertial force along this coordinate is $m\left(d^{2} x_{i} / d t^{2}\right) \delta x_{i}$. Adding the work of all bodies and all Cartesian coordinates to the left hand side of 43 ,

$$
m \sum_{i} \frac{d^{2} x_{i}}{d t^{2}} \delta x_{i}+\sum_{i} Q_{i} \delta q_{i}=0,
$$

which is the analytical expression of the d'Alembert principle according to Lagrange 4]. Lagrange remarks that the differential signalized by $\delta$, called variation, describes arbitrary increments or decrements and should be distinguished from the usual differential, signalized by $d$, which describes increments or decrements caused by the actual motion of the body.

\subsection{Equations of Lagrange}

The equations of motion were obtained by Lagrange from the principle expressed by equation (44) as follows. One starts by performing a change of variables from $x_{i}$ to new variables $\xi_{j}$, not necessarily Cartesian. The relation 
between the differentials is

$$
d x_{i}=\sum_{j} A_{i j} d \xi_{j}
$$

where $A_{i j}=\partial x_{i} / \partial \xi_{j}$ are functions of the new variables $\xi_{j}$, and an analogous relation holds between the variations

$$
\delta x_{i}=\sum_{j} A_{i j} \delta \xi_{j}
$$

The first relation gives

$$
d^{2} x_{i}=\sum_{j}\left(d A_{i j}\right) d \xi_{j}+\sum_{j} A_{i j} d^{2} \xi_{j}
$$

From the last two relations one obtains

$\sum_{i} d^{2} x_{i} \delta x_{i}=\frac{1}{2} \sum_{j k}\left(d B_{j k}\right) d \xi_{j} \delta \xi_{k}+\sum_{j k} B_{j k} d^{2} \xi_{j} \delta \xi_{k}$

where

$$
B_{j k}=\sum_{i} A_{i j} A_{i k}
$$

which depends on the new variables $\xi_{j}$ only. Defining the quantity

$$
\alpha=\frac{1}{2} \sum_{i} d x_{i}^{2}=\frac{1}{2} \sum_{j k} B_{j k} d \xi_{j} d \xi_{k},
$$

the following relations are obtained

$$
\begin{aligned}
& \sum_{k} d\left(\frac{\partial \alpha}{\partial d \xi_{k}}\right) \delta \xi_{k}=\sum_{j k}\left(d B_{j k}\right) d \xi_{j} \delta \xi_{k} \\
& +\sum_{j k} B_{j k} d^{2} \xi_{j} \delta \xi_{k}
\end{aligned}
$$

and

$$
\begin{aligned}
& \sum_{i}\left(\frac{\partial \alpha}{\partial \xi_{i}}\right) \delta \xi_{i}=\frac{1}{2} \sum_{j k}\left(\delta B_{j k}\right) d \xi_{j} d \xi_{k} \\
& =\frac{1}{2} \sum_{j k}\left(d B_{j k}\right) d \xi_{j} \delta \xi_{k} .
\end{aligned}
$$

Comparing (48) with (51) and 52 one reaches the result

$$
\sum_{i} d^{2} x_{i} \delta x_{i}=\sum_{k}\left(d \frac{\partial \alpha}{\partial d \xi_{k}}-\frac{\partial \alpha}{\partial \xi_{k}}\right) \delta \xi_{k} .
$$

The crucial step in the derivation of equation (53) is found in the second equality of equation $(52)$. To show its validity it suffices to determine $\delta\left(d x_{i}\right)$ from 45 and $d\left(\delta x_{i}\right)$ from (46). Since these two quantities are equal, the following relation is obtained

$$
\sum_{j} \delta A_{i j} d \xi_{j}=\sum_{j} d A_{i j} \delta \xi_{j}
$$

where the equality $\delta\left(d \xi_{j}\right)=d\left(\delta \xi_{j}\right)$ has taken into account. Using this result and the relation 49 between $B_{i j}$ and
$A_{i j}$, it is straightforward to reach the second equality of equation 52 .

Lagrange considers next the case where the forces $Q_{i}$ in equation 44 are such that

$$
d V=\sum_{i} Q_{i} d q_{i}
$$

is a complete differential and $V$ will be a function of $q_{i}$. If $V$ is expressed in terms of the variables $\xi_{i}$, we may write

$$
\delta V=\sum_{i} \frac{\partial V}{\partial \xi_{i}} \delta \xi_{i}
$$

The replacement of this expression and the expression (53) in equation (44), yields the result

$$
\sum_{i}\left(d \frac{\partial T}{\partial d \xi_{i}}-\frac{\partial T}{\partial \xi_{i}}+\frac{\partial V}{\partial \xi_{i}}\right) \delta \xi_{i}
$$

where

$$
T=\frac{m}{2} \sum_{i} \frac{d x_{i}^{2}}{d t^{2}}
$$

Lagrange concludes that if the variables $\xi_{i}$ are independent, each coefficient of $\delta \xi_{i}$ will vanish, that is,

$$
d \frac{\partial T}{\partial d \xi_{i}}-\frac{\partial T}{\partial \xi_{i}}+\frac{\partial V}{\partial \xi_{i}}=0
$$

which are the Lagrange equations of motion.

\section{Thermodynamics}

Thermodynamics emerged around the middle of the nineteenth century and presented two changes in the way the heat was conceived [1]. The first was the recognition that heat should be understood as a form of work, which lead to the law of conservation of energy. The second was the way in which heat was transformed in mechanical work, which allowed Clausius to define entropy and lead to the law of the increase in entropy.

The heat absorbed minus the work performed by a system along a thermodynamic process is equal to the increase in the internal energy, and is independent of the trajectory connecting the the final and initial states. The energy is a state function which means to say that differential $d Q-d W$ is a exact differential. In his first paper on thermodynamics [41], Clausius showed that $d Q-d W$ is an exact differential by the use of the equivalence between heat and mechanical work.

Clausius starts his reasoning by assuming that a small quantity $d Q$ of heat exchanged when the volume $V$ and temperature $T$ of a gas changes by $d V$ and $d T$ is given by

$$
d Q=M d V+N d T,
$$

where $M$ and $N$ are functions of $V$ and $T$. Then he considers the total heat exchanged in a clockwise cycle. 


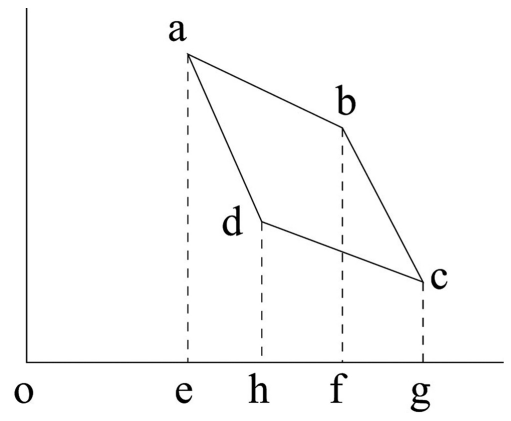

Figure 4: A small Carnot cycle in the pressure-volume diagram. The lines $a b$ and $d c$ are isotherms whereas the lines $a d$ and $b c$ are adiabatics, and $e f=d V, f g=d V_{3}$, eh $=d V_{2}$, and $h g=d V_{1}$. The figure is based on figure 2 of reference [41].

Considering that the cycle is small Clausius argues that the heat exchanged is

$$
\left(\frac{\partial M}{\partial T}-\frac{\partial N}{\partial V}\right) d V d T
$$

which he says is a second order differential.

To reach result (61), Clausius argues as follows. Along the isotherms of the small cycle shown in figure 4

$$
d Q=M d V, \quad d Q_{1}=M_{1} d V_{1},
$$

and along the adiabatic lines

$$
M d V_{2}-N d T=0, \quad M_{2} d V_{3}-N_{2} d T=0 .
$$

The last two equations together with the relation $d V+$ $d V_{3}=d V_{2}+d V_{1}$ allows us to write

$$
d V_{1}=d V+\left(\frac{N_{2}}{M_{2}}-\frac{N}{M}\right) d T
$$

The quantities $M_{1}$ and $M_{2}$ are related to $M$ by

$$
\begin{gathered}
M_{1}=M+\frac{\partial M}{\partial V} d V_{2}-\frac{\partial M}{\partial T} d T, \\
M_{2}=M+\frac{\partial M}{\partial V} d V,
\end{gathered}
$$

and $N_{2}$ is related to $N$ by

$$
N_{2}=N+\frac{\partial N}{\partial V} d V
$$

From these relations we find

$$
\begin{gathered}
M_{1}=M+\left(\frac{\partial M}{\partial V} \frac{N}{M}-\frac{\partial M}{\partial T}\right) d T \\
d V_{1}=d V+\frac{1}{M}\left(\frac{\partial N}{\partial V}-\frac{\partial M}{\partial V} \frac{N}{M}\right) d V d T .
\end{gathered}
$$

These two results give

$$
M_{1} d V_{1}=M d V+\left(\frac{\partial N}{\partial V}-\frac{\partial M}{\partial T}\right) d V d T
$$

The subtraction of $d Q_{1}=M_{1} d V_{1}$ from $d Q=M d V$ gives the result 61.

An analogous result for the net work was obtained by Clausius. Starting from the expression $d W=p d V$, where $p$ is the pressure of the gas, he finds

$$
\left(\frac{\partial p}{\partial T}\right) d V d T
$$

for the net work performed by the gas in a small cycle. We remark that, in his paper of 1850 , one finds $R d V d T / V$ instead of 71 because he used the ideal gas equation $p=R T / V$ [41]. However in a comment to this paper $[2$ he writes the general expression (71).

Clausius carried out an original derivation of results (61) and (71), but they can be understood as a direct application of a theorem formulated by Cauchy in 1846 [42]. According to this theorem of Cauchy, the contour integral of a region in a plane is related to an integral over this region as follows

$$
\oint(X d x+Y d y)=\int\left(\frac{\partial Y}{\partial x}-\frac{\partial X}{\partial y}\right) d x d y .
$$

In the particular case where $X d x+Y d y$ is an exact differential, Cauchy reminds that $\partial Y / \partial x=\partial X / \partial y$ and the integral vanishes.

Next Clausius uses the law of Mayer and Joule according to which the work is always transformed in the same quantity of heat. If a certain quantity of work $W$ is dissipated, the quantity of heat generated $q=A W$ where $A$ expresses the equivalent of heat in terms of mechanical work. The reciprocal of $A$ is the mechanical equivalent of heat. Here, we use a practice that became common in thermodynamics which is to express heat in terms of mechanical unit which is equivalent to say that a quantity if heat $Q$ is related to $q$ by $Q=q / A$. Using this procedure, the law of Mayer and Joule becomes $Q=W$, which results in the equality of expressions 61 and (71),

$$
\frac{\partial M}{\partial T}-\frac{\partial N}{\partial V}=\frac{\partial p}{\partial T}
$$

Since $\partial p / \partial T$ is nonzero, the left-hand side of $\sqrt{73}$ is nonzero and $d Q$ given by 60 cannot be an exact differential, concludes Clausius.

If we define the quantity $c=M-p$, it follows from 73) that $\partial c / \partial T=\partial N / \partial V$, which is the condition for $c d V+N d T$ being an exact differential. Clausius calls this differential $d U$ and writes

$$
d U=(M-p) d V+N d T .
$$

As it is, equation (74) cannot be integrated unless one knows $M, N$ and $p$ as a function of $V$ and $T$. This was accomplished by Clausius for an ideal gas. In addition to the equation of state, Clausius relies on another law which he says is valid as much a the equation of state. When an ideal gas expands isothermally, the heat absorbed is entirely transformed into work, from which follows that 
$d Q=d W=p d V$ or $d U=0$ along an isotherm. In an equivalent form $(\partial U / \partial V)_{T}=0$, that is, the function $U$ is independent of $V$, depending only on $T$.

Recalling that $d Q=M d V+N d T$, equation (74) is written in the form $d Q=d U+p d V$ and we see that the heat capacity at constant volume is

$$
C_{v}=\left(\frac{d Q}{d T}\right)_{V}=C
$$

where $C=d U / d T$, and depends only on $T$, and that the heat capacity at constant pressure is

$$
C_{p}=\left(\frac{d Q}{d T}\right)_{p}=C+R,
$$

from which follows $C_{p}=C_{v}+R$, that is, the difference in the heat capacities of ideal gas is a constant.

The way in which heat is transformed in mechanical work was the main subject of research on heat carried out by Carnot. His investigations lead him to the following fundamental principle. When a system undergoes a cyclic process composed by two isothermal and two adiabatic processes the ratio of the work produced and the heat depends only on the two temperatures. In this principle of Carnot, heat was understood as a conserved quantity which in this case descends from a high temperature to a low temperature. Clausius modified this principle by replacing heat by heat absorbed. In addition he uses the law of Mayer and Joule to state that the work $W$ is the heat absorbed $Q_{1}$ by the system at high temperature minus the heat $Q_{2}$ released by the system at low temperature, or more precisely, $Q=Q_{1}-Q_{2}$.

The modified principle of Carnot was written by Clausius in the form $Q_{1} / T_{1}=Q_{2} / T_{2}$ where $T_{1}$ and $T_{2}$ are the absolute temperatures corresponding to the two isotherms. The generalization of this expression for any cycle lead Clausius to the result that $d Q / T$ is a exact differential 43 . Later on, he wrote $d S=d Q / T$ and called $S$ the entropy [44]. The infinitesimal heat absorbed by a system in equilibrium becomes related to the differential of entropy by $d Q=T d S$ and

$$
d U=T d S-p d V
$$

which is the conservation of energy in differential form, where all differentials involved are exact differentials.

\section{Conclusion}

We have analyzed the role of exact and inexact differentials in the early developments of mechanics and thermodynamics. We have also examined the evolution of differential calculus in relation to the concepts related to exact and inexact differentials. Euler introduced the concept of integrating factor, which he used to solve an ordinary linear differential equation of the first-order. Euler also found the condition for a complete differential by examining the differential of a function of two variables. In an independent way, Clairaut also reached the same condition. When this condition is not fulfilled the differential is incomplete or inexact. In the analytical treatment of mechanics, Lagrange considered forces whose differential work is an exact differential, in which case it is possible to define a work function and reach the conservation of energy.

When the differential is inexact, it is possible to transform it into and an exact differential as long as an integrating factor can found. The fundamental law of equilibrium thermodynamics introduced by Clausius stating that $d Q / T=d S$ can equally be formulated by declaring that $1 / T$ is an integrating factor related to the inexact differential $d Q$. The law of conservation of energy was also written by Clausius in terms of exact differentials. The process of finding exact differentials was a fundamental procedure which allowed the formulation of thermodynamics in terms of state functions such as energy and entropy.

\section{References}

[1] M.J. Oliveira, Braz. J. Phys. 48, 299 (2018).

[2] R. Clausius, Abhandlungen über die Mechanische Wärmetheorie (Vieweg und Sohn, Braunschweig, 1864).

[3] R. Dugas, A History of Mechanics (Routledge and Kegan Paul, London, 1955).

[4] J.L. Lagrange Méchanique Analitique (Veuve Desaint, Paris, 1788).

[5] C.B. Boyer, The History of the Calculus and its Conceptual Development (Dover, New York, 1959).

[6] F. Cajori, The American Mathematical Monthly 26, 15 (1919).

[7] F. Cajori, A History of Mathematical Notations, Volume II (Open Court, Chicago, 1929).

[8] D.J. Struik (ed.), A Source Book in Mathematics, 12001800 (Princeton University Press, Princeton, 1986).

[9] W. Dunham, The Calculus Gallery, Masterpieces from Newton to Lebesgue (Princeton University Press, Princeton, 2005).

[10] J.S. Bardi, The Calculus Wars, Newton, Leibniz, and the Greatest Mathematical Clash of All Time (High Stakes, Ebbw Vale, 2006).

[11] D.T. Whiteside, The Mathematical Works of Isaac Newton (Johson Reprint Corporation, New York, 1964), v. 1.

[12] G.W. Leibniz, Acta Eruditorum, Octobris 1684, p. 467.

[13] G.W. Leibniz, Acta Eruditorum, Junii 1686, p. 292.

[14] H. Eves, An Introduction to the History of Mathematics (Holt, Rinehart and Winston, New York, 1969), 3rd. ed.

[15] G.F.A. de l'Hôpital, Analyse des Infiniment Petits pour l'Intelligence de Lignes Courbes (Imprimerie Royale, Paris, 1696).

[16] L. Euler, Commentarii Academiae Scientiarum Imperialis Petropolitanae 3, 124 (1732).

[17] L. Euler, Commentarii Academiae Scientiarum Imperialis Petropolitanae 7, 174 (1740).

[18] L. Euler, Institutiones Calculi Differentialis (Academiae Imperialis Scientiarum, Petropolitanae, 1755).

[19] A.C. Clairaut, Memoires de l'Académie Royale de Sciences, année 1740, 293 (1742). 
[20] A.C. Clairaut, Theorie de la Figure de la Terre Tirée des Principes de l'Hidrodynamique (David Fils, Paris, 1743).

[21] J.R. d'Alembert, in: Encyclopédie, publié par M. Diderot et J.R. d'Alembert (Briasson, David, Le Breton, Durand, Paris, 1754), Tome Quatrieme, p. 985.

[22] J.L. Lagrange, Nouveaux Mémoires de l'Académie Royale de Sciences et Belles-Lettres, année 1772, p. 185 (1774).

[23] J.L. Lagrange, Théorie des Fonctions Analytiques (Imprimerie de la République, Paris, 1797).

[24] B. Taylor, Methodus Incrementorum Directa et Inversa (Pearsonianis, London, 1715).

[25] A.L. Cauchy, Résumé de Leçons Données a l'École Royale Polythechnique, sur le Calcul Infinitésimal (Imprimerie Royale, Paris, 1823).

[26] J.V. Grabiner, American Mathematical Monthly 90, 185 (1983).

[27] J.L. Fourier Théorie Analytique de la Chaleur (Firmin Didot, Paris, 1822).

[28] P.G.L. Dirichlet, Repertorium der Physik 1, 152 (1837).

[29] I. Newton, Philosophiae Naturalis Principia Mathematica (S. Pepys, London, 1687).

[30] I. Newton, The Mathematical Principles of Natural Philosophy (Benjamin Motte, London, 1729), 2 vols.

[31] G. Galilei, Discorsi e Dimostrazioni Matematiche intorno à due nuove Scienze (Elsevirii, Leida, 1638).

[32] G. Galilei, Dialogues Concerning two New Sciences (MacMillan, New York, 1914).

[33] A.E. Bell, Christian Huygens and the Development of Science in the Seventeenth Century (Arnold, London, 1949).

[34] C. Huygens, Horologium Oscillatorium sive de Motu Pendulorum ad Horologia Aptato Demonstrationes Geometricae (F. Muguet, Paris, 1673).

[35] Johann Bernoulli, Acta Eruditorum, Junii 1696, p. 264.

[36] Johann Bernoulli, Acta Eruditorum, Maji 1697, p. 206.

[37] G.W. Leibniz, Nouvelles de la Republique des Lettres, Septembre 1687, p. 952.

[38] Jacob Bernoulli, Acta Eruditorum, Maji 1690, p. 219.

[39] P. Varignon, Nouvelle Mecanique ou Statique (Jombert, Paris, 1725), v. 2.

[40] J. le Rond d'Alembert, Traité de Dynamique (David, Paris, 1743).

[41] R. Clausius, Annalen der Physik und Chemie 79, 368 (1850).

[42] A. Cauchy, Comptes Rendus Hebdomadaires des Séances de l'Académie des Sciences 23, 251 (1846).

[43] R. Clausius, Annalen der Physik und Chemie 93, 481 (1854).

[44] R. Clausius, Annalen der Physik und Chemie 125, 353 (1865). 